\title{
GW23-e0581 THE SAFETY AND EFFICACY OF DUAL-AXIS ROTATIONAL CORONARY ANGIOGRAPHY IN THE DIAGNOSIS OF CORONARY ARTERY DISEASE
}

doi:10.1136/heartjnl-2012-302920l.16

Yang Shengli, Liu Huiliang, Wang Lei, Yang Shengli. Department of Cardiology, The General Hospital of Chinese People's Armed Police Forces

Objectives Standard coronary angiography (SA) is performed in multiple stationary views at different angles around the patient to evaluate the severity and location of the lesions. The procedure exposes patients to the potential health risks from contrast and radiation exposure. During dual axis rotations the x-ray system rotates with trails pre-programmed around the patient with a single injection. This study evaluates the efficacy and safety of Dual-axis rotational coronary angiography (DARCA) (X-per Swing) in the diagnosis of coronary artery disease.

Methods From March to July in 2010, consecutive 79 patients undergoing diagnostic coronary angiography were randomised to either standard angiography group $(n=39)$ or X-per Swing angiography group $(n=40)$. We measured the quantity of contrast utilised and radiation exposure. The number of additional angiography needed to be performed was used to evaluate and compare the efficacy of two methods.

Results Both groups were successfully completed angiography. All angiograms were reviewed for CAD screening efficacy by two independent invasive cardiologists. There was a $44 \%$ reduction in contrast utilisation in the X-per Swing group compared to the standard group $(29.28 \pm 5.06 \mathrm{ml}$ vs $52.02 \pm 12.05 \mathrm{ml}, \mathrm{p}<0.001)$. Additionally, there was a $50 \%$ reduction in radiation exposure in the X-per Swing group compared to the standard group $\left(6900 \pm 3443.03 \mathrm{mGycm}^{2}\right.$ vs 16857 $\left.\pm 8584.68 \mathrm{mGycm}^{2}, \mathrm{p}<0.001\right)$. Neither arrhythmia nor chest pain differed in both groups. X-per Swing can provides a significant reduction of contrast and radiation exposure while maintaining comparable diagnostic accuracy and safety. With an auto-inject system (ACIST CMS2000) that can autoinject contrast by interlinkage, operator can stay far away from $\mathrm{x}$-ray tube, which enable the $\mathrm{x}$-ray exposure to be extremely reduced.

Conclusions X-per Swing (DARCA) represents a new angiographic technique which is equivalent in terms of image quality and is associated with less contrast use, radiation exposure and procedural time than SA. 